\title{
Angular Correlations in Three-Jet Production and Jet Substructure in Neutral Current Deep Inelastic Scattering at HERA
}

\author{
Elias Ron, on behalf of the ZEUS collaboration \\ Universidad Autonoma de Madrid
}

\begin{abstract}
Two analyses concerning jet production in ep collisions at a center-of-mass energy of $\sqrt{s}=318 \mathrm{GeV}$ are presented. Firstly, measurements of three-jet angular correlations between the three jets in the final state and the beam direction are investigated to separate the contributions from the different colour configurations. Secondly, measured normalised cross sections on subjet observables were used to study the pattern of parton radiation. In both analyses, jets are produced in Neutral Current (NC) deep inelastic scattering (DIS), with a required virtuality of the interaction boson $Q^{2}>125 \mathrm{GeV}^{2}$. A luminosity of $81.7 \mathrm{pb}^{-1}$, taken by the the ZEUS detector at HERA during the years 1998-2000, was used.
\end{abstract}

\section{Angular correlations in three-jet production}

\subsection{Introduction}

The self-coupling of the gluons is an essential feature of Quantum Chromodynamics (QCD). This is interpreted as a consequence of the SU(3), a non-Abelian group, being the underlying gauge symmetry group describing the strong interactions. The group structure is characterised in the perturbative expansion of the cross section through the colour factors, which depend on the underlying symmetry group. At leading order in the perturbative expansion, the cross section for three-jet production in ep scattering can be expressed as a sum of different colour configurations [2]. The partonic cross sections are:

$$
\sigma_{e p \rightarrow 3 \text { jets }}=C_{F}^{2} \cdot \sigma_{A}+C_{F} C_{A} \cdot \sigma_{B}+C_{F} T_{F} \cdot \sigma_{C}+T_{F} C_{A} \cdot \sigma_{D}
$$

In $\mathrm{QCD}$, the corresponding values for the different colour configurations are $C_{F}=4 / 3$ for the configuration that includes the $q \rightarrow q g$ vertex, $C_{A}=3$ for the configuration which includes the triple-gluon vertex and $T_{F}=1 / 2$ for the one containing the $q \rightarrow q \bar{q}$ vertex. However, for an Abelian theory like QED, $C_{F}=1, C_{A}=0, T_{F}=3$. Thus, variables which are sensitive to contributions from different colour configurations are sensitive to the underlying symmetry group of the theory. The jet angular correlation variables used in the analysis are:

- $\alpha_{23}$, defined as the angle between the two lowest transverse energy jets.

- $\beta_{K S W}$, defined as

$$
\cos \left(\beta_{K S W}\right)=\cos \left[\frac{1}{2}\left(\angle\left[\left(\overrightarrow{p_{1}} \times \overrightarrow{p_{3}}\right),\left(\overrightarrow{p_{2}} \times \overrightarrow{p_{B}}\right)\right]+\angle\left[\left(\overrightarrow{p_{1}} \times \overrightarrow{p_{B}}\right),\left(\overrightarrow{p_{2}} \times \overrightarrow{p_{3}}\right)\right]\right)\right],
$$

where $\overrightarrow{p_{i}}, i=1, \ldots, 3$ is the momentum of jet $i$ and $\overrightarrow{p_{B}}$ is a unit vector in the direction of the beam; the jets are ordered according to decreasing transverse energy;

- $\eta_{\max }^{\text {jet }}$, the maximum pseudorapidity of the three jets with highest transverse energy. 

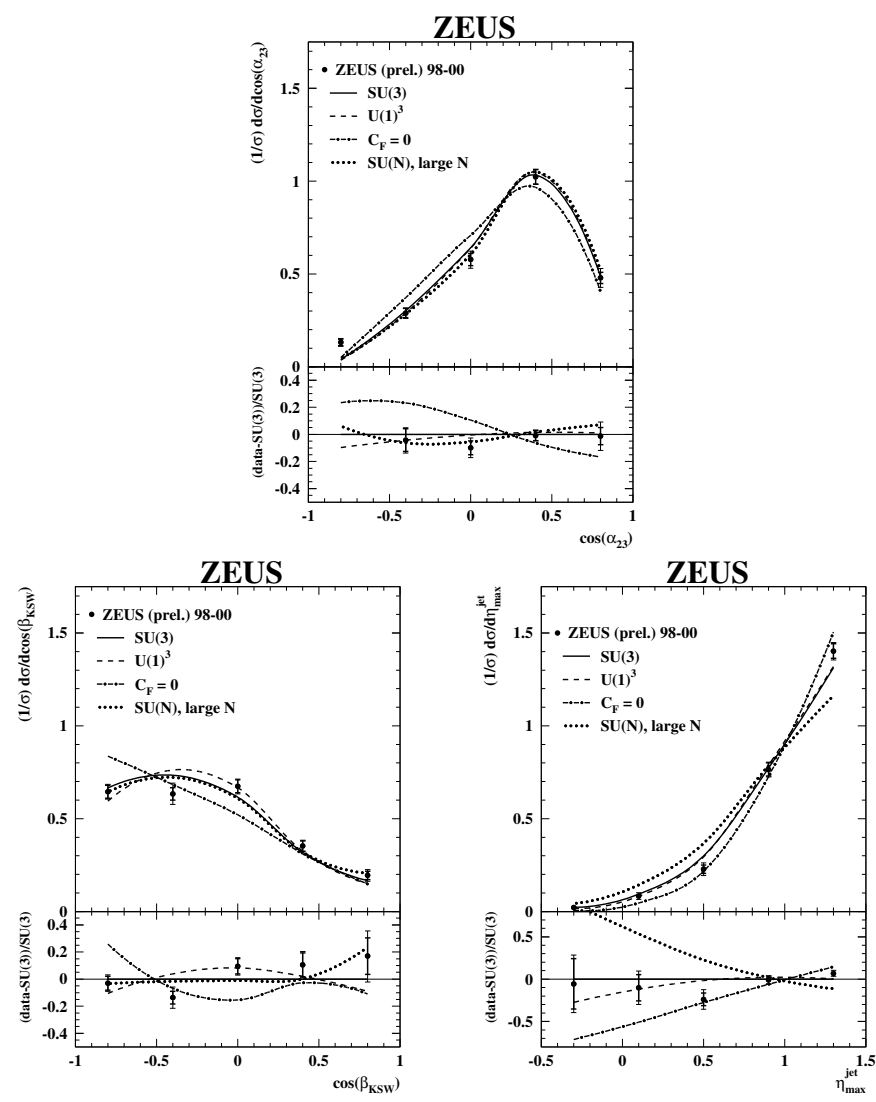

Figure 1: Measured differential cross sections as functions of the angular variables.

\subsection{Results}

Figure 1 shows the distribution of the normalised differential cross sections as functions of the angular variables. The data (black dots) are compared to four different predictions based on different underlying gauge symmetry groups, the non-Abelian SU(3), the Abelian group $U(1)^{3}, \mathrm{SU}(\mathrm{N})$ for large $\mathrm{N}$ and a situation with $C_{F}=0, T_{F}=1 / 2$ and $C_{A}=3$. The inner error bar corresponds to the statistical error and the outer to the addition in quadrature of the statistical and systematical uncertainties. The theoretical predictions are obtained at leading-order (LO) with the program DISENT [3]. The calculations for each colour configuration are obtained separately and then the predictions for different underlying symmetry groups are obtained by multiplying by the corresponding colour factors. The data are found to be consistent with the admixture of colour configurations based on $\mathrm{SU}(3)$, as predicted by QCD. The normalised differential cross section as function of $\eta_{\max }^{\text {jet }}$ disfavours an underlying symmetry group based on $\mathrm{SU}(\mathrm{N})$ for large $\mathrm{N}$ and also the situation with $C_{F}=0$. This variable also provides sensitivity to distinguish the predictions based on $\mathrm{SU}(3)$ and the Abelian group $U(1)^{3}$. However, the experimental precision is not yet sufficient to do so. 


\section{Jet substructure}

\section{$2.1 \quad$ Introduction}

The investigation of the internal structure of jets gives insight into the transition between a parton in a hard process and the experimentally observable jet of hadrons. At high transverse energy of the jets, $E_{T}^{\text {jet }}$ the effects of fragmentation become negligible and the jet substructure can be calculated perturbatively. The $k_{T}$ cluster algorithm [4] was used to define jets in the final state. Subjets were resolved within a jet by considering all particles associated with the jet and repeating the application of the jet finding algorithm until, for every pair of particles $i$ and $j$ the quantity:

$$
d_{i j}=\min \left(E_{T, i}, E_{T, j}\right)^{2}\left[\left(\eta_{i}-\eta_{j}\right)^{2}+\left(\phi_{i}-\phi_{j}\right)^{2}\right]
$$

was greater than $d_{\text {cut }}=y_{\text {cut }}\left(E_{T}^{\text {jet }}\right)^{2}$. The remaining clusters are called subjets. Subjet multiplicity it then defined as the number of subjets resolved within the jet, and depends on the value of the resolution parameter $d_{\text {cut }}$. The jet sample in this analysis consists of those jets with exactly two subjets at a $y_{\text {cut }}=0.05$. The pattern of QCD radiation from a primary parton has been studied by measing normalised cross sections as a function of the following subjet observables: the ratio between the subjet transverse energy and that of the jet, $E_{T}^{\mathrm{sbj}} / E_{T}^{\mathrm{jet}}$, the difference between the subjet pseudorapidity (azimuth) and that of the jet, $\eta^{\mathrm{sbj}}-\eta^{\mathrm{jet}}\left(\left|\phi^{\mathrm{sbj}}-\phi^{\mathrm{jet}}\right|\right)$ and $\alpha^{\mathrm{sbj}}$, the angle, as viewed from the jet center, between the highest transverse energy subjet and the beam line in the pseudorapidity-azimuth plane. The calculations at $O\left(\alpha_{s}\right)$ and $O\left(\alpha_{s}^{2}\right)$ QCD calculations are used to compare with the data are based on the program DISENT.

\subsection{Results}

Figure shows the normalised differential cross sections as function of the variables described above. The black dots are the data, the inner error bar corresponds to the statistical uncertainty and the outer error bar to the addition in quadrature of the statistical and systematical uncertainties.

The distribution in $E_{T}^{\text {jet }} / E^{T}$, which is symmetric by construction, shows that the two subjets tend to have similar transverse energies. The distribution in $\eta^{\mathrm{sbj}}-\eta^{\text {jet }}$ shows an asymmetric two peak structure. The dip in the middle is a consequence of the inability to resolve subjets which are very close to each other. The distribution shows that the two subjets tend to be near to each other. The normalised differential cross section as function of $\left|\phi^{\text {sbj }}-\phi^{\text {jet }}\right|$ also indicates that subjets tend to be close to each other, and the dip at low values of the variable is also a consequence of resolution limitations when they are very near. As can be seen, the cross section as function of $\alpha^{\text {sbj }}$ grows as $\alpha^{\text {sbj }}$ grows, which indicates that the highest transverse energy subjet tends to be in the rear part of the jet. This feature, together with the fact that the highest $E_{T}$ subjet tends to be closer to the jet center than the other subjet, is compatible with the asymmetry observed in the $\eta^{\text {sbj }}-\eta^{\text {jet }}$ distribution. 

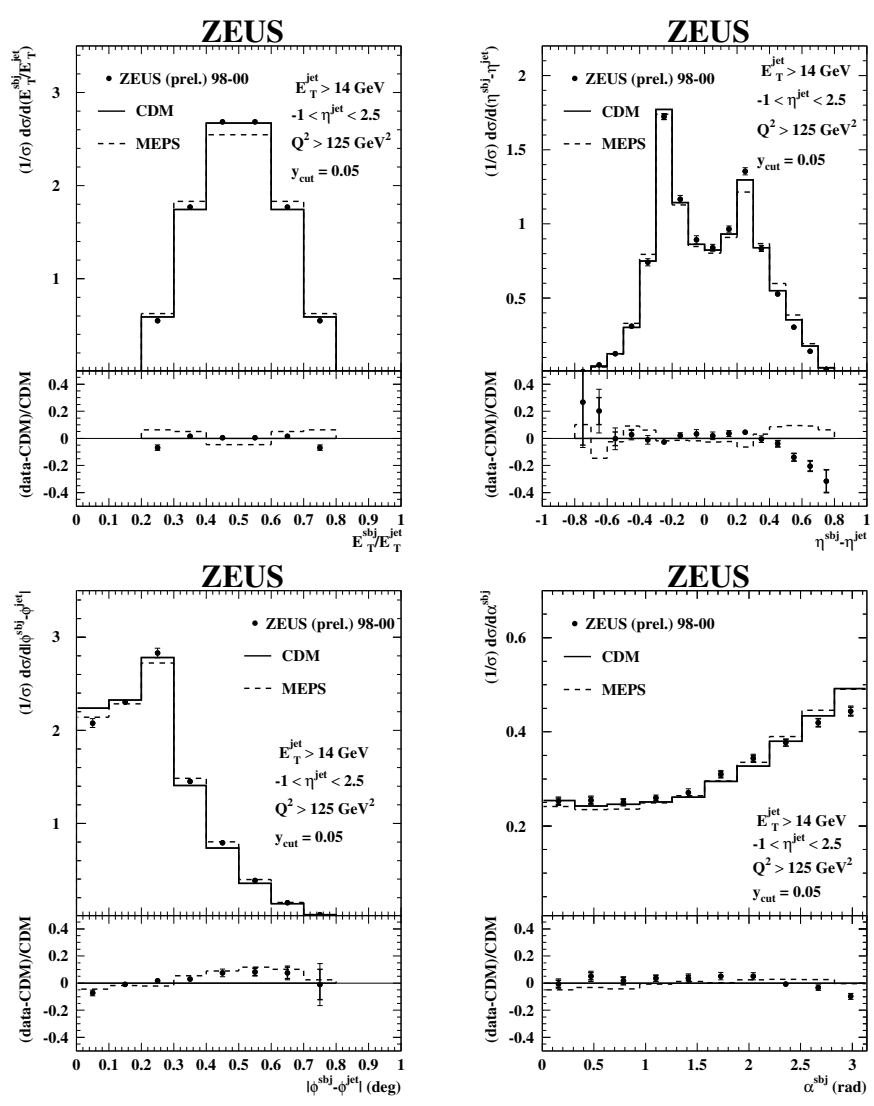

Figure 2: Measured differential cross sections as functions of a) $E_{T}^{\text {jet }} / E^{T}$, b) $\eta^{\text {sbj }}-\eta^{\text {jet }}$, c) $\left|\phi^{\text {sbj }}-\phi^{\text {jet }}\right|$ and d) $\alpha^{\text {sbj }}$.

\section{References}

[1] Slides:

http://indico . cern. $\mathrm{ch} /$ contributionDisplay . py? contribId=225\&sessionId=6\&conf Id $=9499$

[2] P. Aureche et al., Nucl. Phys. Phys. B 268, 553 (1987)

[3] S.Catani and M.H. Seymour, Nucl. Phys. B 485, 291 (1997).

[4] S.Catani et al. Nucl.Phys. B 406, 187(1993). 